\title{
Identification of $F B N 1$ gene mutations in patients with ectopia lentis and marfanoid habitus
}

\author{
P Comeglio, A L Evans, G Brice, R J Cooling, A H Child
}

Br J Ophthalmol 2002;86:1359-1362

Background: Marfan syndrome (MFS), inherited as an autosomal dominant trait, typically affects the cardiovascular, skeletal, and ocular systems. Ectopia lentis (EL) is a clinical manifestation of MFS, with stretching or disruption of the lenticular zonular filaments, leading to displacement of the lenses. EL, with or without minor skeletal changes, exists as an independent autosomal dominant phenotype linked to the same FBN1 locus.

Methods: A consecutive series of 11 patients, affected predominantly by $\mathrm{EL}$, was analysed for $\mathrm{FBN} 1$ mutations using PCR, SSCA, and sequencing.

Results: Six mutations were identified, of which three are novel and one is recurrent in two patients, thus establishing a mutation incidence in this group of $7 / 11(63 \%)$.

Conclusion: The FBN 1 variants reported are clustered in the first 15 exons of the gene, while FBN1 mutations reported in the literature are distributed throughout the entire length of the gene. A different type of $F B N 1$ mutation presents in this group of patients, compared with MFS, with arginine to cysteine substitutions appearing frequently.

$\mathrm{E}^{2}$ ctopia lentis (EL, MIM 129600) is an autosomal dominant connective tissue disorder, in which the lenses tend to be dislocated upward and the zonular filaments are stretched or discontinuous. ${ }^{1}$ EL belongs to the clinical spectrum of diseases related to Marfan syndrome (MFS, MIM 154700), and it presents with some of the skeletal, but not serious cardiovascular, features of MFS. ${ }^{2}$ MFS is an autosomal dominant connective tissue disorder, characterised by variable phenotypic manifestations mainly in cardiovascular, skeletal, and ocular systems. ${ }^{3}$

Co-localisation of MFS and EL loci and the gene FBN1 (MIM 134797) has been demonstrated. ${ }^{2}{ }^{4}$ The genomic copy of the FBNl gene is $235 \mathrm{~kb}$ and encodes for fibrillin-1, a secreted $350 \mathrm{kDa}$ glycoprotein, major structural component of the elastin associated $10-12 \mathrm{~nm}$ microfibrils, ${ }^{56}$ which are the sole structural element visible by conventional electron microscopy in the suspensory ligaments of the lens. Fibrillin- 1 is mainly composed of cysteine rich epidermal growth factor (EGF)-like domains, most of them with a calcium binding consensus sequence (cbEGF-like). ${ }^{15}$ Mutations at the calcium binding sites, and cysteine substitutions seem to affect the structural function of fibrillin- $1 .{ }^{1}$

FBN1 mutations have been characterised in patients affected by type I fibrillinopathies, ${ }^{7-9}$ which include MFS, MASS syndrome (MIM 604308), EL, Shprintzen-Goldberg syndrome (MIM 182212), isolated skeletal features of MFS, and thoracic aortic aneurysms. The identified mutations are distributed throughout the FBNI gene, with limited evidence of genotype-phenotype correlation. ${ }^{8}$ In particular, only the cluster of exons 24 to 32 is linked with a severe form of MFS, neonatal MFS. ${ }^{1} 1011$
To date, only four FBN1 mutations have been reported in patients affected by predominant EL, the less severe form of the disease continuum. ${ }^{12-14}$ Clinically, differential diagnosis and overall management of EL patients is problematical. Many patients are never referred for cardiological assessment to rule out MFS.

In this study we characterised the incidence and class of FBN1 mutations in a group of 11 consecutive unrelated British patients affected predominantly by EL.

We identified six causative or putative mutations in the FBN1 gene, three of which have not been previously reported, and one of which is recurrent in two patients, thus establishing an FBN1 mutation incidence of 63\% (7/11) in the patients studied, not including three $F B N 1$ variants classified as polymorphisms.

\section{SUBJECTS AND METHODS}

We investigated 11 consecutive patients (seven men; four women) with predominant EL, eight of whom had a family history of dominantly inherited lens dislocation, and in whom a diagnosis of MFS was excluded according to the current diagnostic criteria. ${ }^{3}$ Physical examinations and investigations are reported in Table 1, and included echocardiography with measurement of aortic root diameter (adult upper limit 39 $\mathrm{mm}$ ), assessment of valve morphology and function, skeletal features, skin extensibility and lumbar striae, assessment of visual acuity, slit lamp and fundus examination. In the absence of a suitable clinical indication, such as severe chronic low back or abdominal pain, magnetic resonance imaging (MRI) for dural ectasia was not performed. Peripheral blood samples were collected, with appropriate informed consent, from probands and available family members.

\section{Mutational analysis}

A set of 65 pairs of primers (Sigma-Genosys, Pampisford, Cambridgeshire, UK) was used for polymerase chain reaction (PCR) amplification of all 65 exons of the FBNl gene. The oligonucleotide sequences were those previously described ${ }^{15}$ or reported in the literature. ${ }^{16}{ }^{17}$ All PCR reagents except primers were supplied by Gibco-BRL Life Technologies (Paisley, UK). The amplifications were performed in a DNA Thermal-Cycler (Hybaid, Ashford, Middlesex, UK) under conditions depending on the exon analysed.

Single strand conformation analysis (SSCA) was carried out as previously described. ${ }^{15}$ Electrophoresis and silver staining chemicals were from Merck (Poole, Dorset, UK) and Sigma-Aldrich (Poole, Dorset, UK).

Sequencing was carried out on an ABI310 using the Big Dye chemistry (Applied Biosystems, Warrington, Cheshire, UK).

Detailed protocols are available on request.

\section{RESULTS}

We analysed 11 patients, whose clinical findings are schematically reported in Table 1 . The six FBN1 mutations, one of which recurred in two patients, are reported in Table 2, together with 
Table 1 Clinical details of 11 consecutive patients affected by predominant EL

\begin{tabular}{|c|c|c|c|c|c|c|c|c|c|c|c|}
\hline & OP & BM & RWT & GB & $\mathrm{JL}$ & NS & MG & Vw & IBP & $M Z$ & SR \\
\hline \multicolumn{12}{|l|}{ Patient } \\
\hline Age (years) & 49 & 41 & 56 & 51 & 40 & 10 & 52 & 65 & 50 & 33 & 32 \\
\hline Sex* ${ }^{*}$ & M & $\mathrm{F}$ & M & M & $\mathrm{F}$ & M & $\mathrm{F}$ & M & $\mathrm{F}$ & M & M \\
\hline Ectopia lentis family history & - & + & + & + & + & + & + & + & + & - & - \\
\hline FBN1 change & $\mathrm{R} 122 \mathrm{C}$ & N164S & R240C & R545C & R545C & S634P & C652Y & A/G 3963 & IVS6 del T -26 & IVS62 $A+8$ to $C$ & - \\
\hline \multicolumn{12}{|l|}{ Skeletal system } \\
\hline Height $(\mathrm{cm})$ & 189 & 163 & 181 & 187 & 170 & 138 & 158 & 184 & 157 & 174 & 185 \\
\hline Arm span $(\mathrm{cm})$ & 187 & 167 & 181 & 189 & 166 & 135 & 157 & 197 & 156 & 181 & 197 \\
\hline $\mathrm{AS} / \mathrm{H} \dagger$ (normal <1.05) & 0.99 & 1.02 & 1 & 1.01 & 0.97 & 0.98 & 0.99 & 1.07 & 0.99 & 1.04 & 1.06 \\
\hline US/LS $\ddagger$ (normal >0.87) & 0.81 & 0.79 & 0.93 & 1.07 & 0.9 & 1.03 & 0.9 & 0.94 & 0.92 & 0.85 & 0.78 \\
\hline Pectus carinatum & - & - & - & - & + & - & - & - & - & - & + \\
\hline Pectur excavatum & - & - & - & + & - & - & - & - & - & - & - \\
\hline Scoliosis & - & - & - & - & - & + & - & + & - & - & + \\
\hline Arachnodactyly & + & - & - & - & - & - & + & - & - & + & - \\
\hline High palate & + & - & - & + & + & + & - & + & - & - & - \\
\hline Joint hypermobility & - & - & + & + & + & + & - & - & + & - & - \\
\hline \multicolumn{12}{|l|}{ Cardiovascular system } \\
\hline Aortic root dimension $(\mathrm{mm})$ & 32 & 28 & 35 & 41 & 32 & 22 & 40 & 39 & 37 & 25 & 40 \\
\hline Mitral valve prolapse & + & - & - & - & - & - & - & - & + & - & + \\
\hline \multicolumn{12}{|l|}{ Ocular system } \\
\hline Lens dislocation & + & + & + & + & + & + & + & + & + & + & + \\
\hline Myopia & + & - & + & - & + & - & + & - & - & - & - \\
\hline Strabismus & - & - & + & - & - & - & - & - & - & - & - \\
\hline Glaucoma & - & - & - & - & - & - & - & + & - & - & - \\
\hline \multicolumn{12}{|l|}{ Other manifestations } \\
\hline Hyperextensible skin & + & - & + & - & - & - & - & - & - & + & + \\
\hline Striae & - & + & + & + & + & - & - & + & - & + & + \\
\hline Hernia & - & - & + & - & - & - & - & + & - & - & - \\
\hline
\end{tabular}

three DNA variants which might represent rare polymorphisms. Several known polymorphisms were identified, without any significant difference in distribution between patients and controls (data not shown). The known polymorphisms and the mutations found were tested in all family members available. Wherever possible to establish, the polymorphisms were not the results of de novo changes in the DNA of patients. The mutations were absent in 160 chromosomes from unrelated controls and in 280 chromosomes from unrelated patients affected by cardiovascular diseases, including thoracic aortic aneurysm and MFS (data not shown). This result led us to exclude the possibility of the mutations identified being polymorphisms.

\section{Clinical summaries}

Patients IBP, VW, and MZ demonstrated only FBNI variants likely to represent polymorphisms, so that no further blood samples were taken from family members. No family members were available for patients OP and JL.

In patient BM the mutation was demonstrated in the proband's younger sister, who has bilateral EL and glaucoma, and in this sister's as yet unaffected 5 year old son. The proband's unaffected older sister demonstrated the mutation, but is clinically unaffected, presumably an example of reduced penetrance, as reported previously in another EL family. ${ }^{12}$ The proband's unaffected mother and brother did not carry the mutation, presumably inherited from the proband's deceased father, who appears to have large eyes in his photographs, although he is not known to have had EL or glaucoma.

The mutation discovered in patient GB was also found in an affected brother but not in the brother's two unaffected adult daughters. Both parents of the proband, including an affected father, died of unrelated causes (cancer) and DNA samples were unavailable.

The echocardiogram of 10 year old patient NS was within normal limits for age. The father of proband NS carries the mutation but does not have dislocated lenses. He demonstrates slight facial asymmetry with simple ears, slight rib sulcus on the left anteriorly, and a non-progressive dilated aortic root (40 mm; upper normal limit $39 \mathrm{~mm}$ ), unchanged at 42 and 45 years, with mild aortic regurgitation. He has slightly lax skin over the elbow and large hands and feet. The proband's brother is unaffected on eye examination and does

Table 2 Results of FBN1 analysis in 11 consecutive patients affected by predominant EL

\begin{tabular}{llllll}
\hline Patient & Mutation site & Nucleotide & Amino acid & Protein domain & References \\
\hline OP & Exon 4 & C364T & R122C & EGF-like No 2 & 13, 18, 19, this report \\
BM & Exon 5 & A491G & N164S & EGF-like No 3 & This report \\
RWT & Exon 6 & C718T & R240C & Hybrid Motif No 1 & 13,14, this report \\
GB & Exon 13 & C1633T & R545C & cbEGF-like No 4 & 13,17, this report \\
JL & Exon 13 & C1633T & R545C & cbEGF-like No 4 & 13, 17, this report \\
NS & Exon 15 & T1900C & S634P & cbEGF-like No 6 & This report \\
MG & Exon 15 & G1955A & C652Y & cbEGF-like No 6 & This report \\
VW & Exon 31 & A3963G & - & cbEGF-like No 1 & 21, this report \\
IBP & Intron 6 & IVS6 del T-26 & - & cbEGF-like No 40 & 22, this report \\
MZ & Intron 62 & IVS62 A+8 to C & - & cbEGF-like No 41 & 14, this report \\
SR & No mutation identified & & & \\
\hline
\end{tabular}




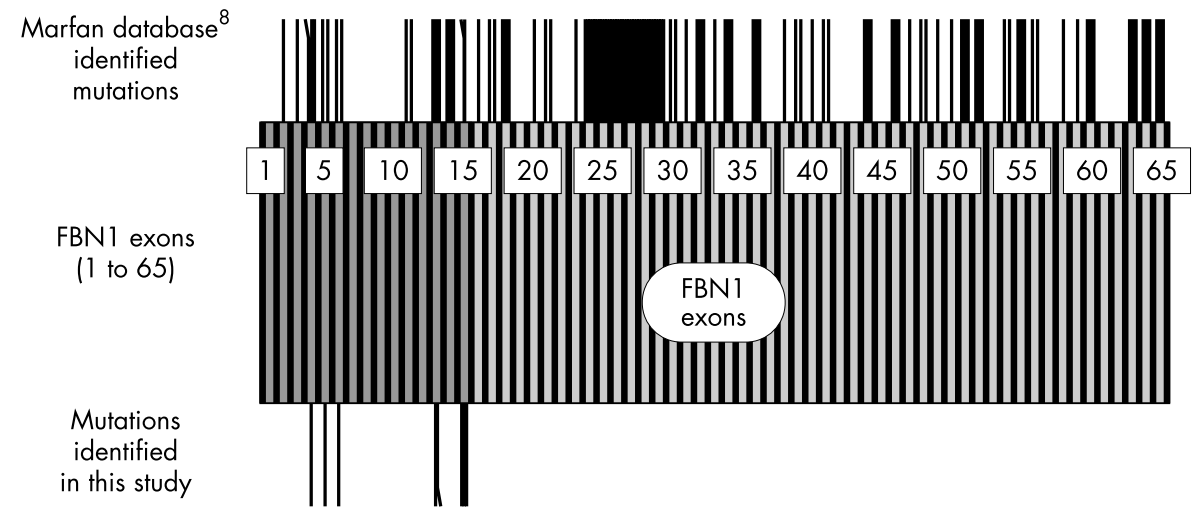

Figure 1 Schematic representation of the FBN1 mutations distribution. At the top are the FBN1 mutations reported in the Marfan database, ${ }^{8}$ while at the bottom are indicated the mutations identified in this study. The first 15 exons of the gene, encoding for the $\mathrm{N}$-terminal domains of the fibrillin-1 protein, are highlighted in dark grey, with the others represented in light grey. The exons are numbered from 1 to 65 .

not carry the mutation. This family demonstrates intrafamilial phenotype variability.

The family of patient MG demonstrates six affected members in three generations, with dominantly inherited EL. The proband's affected mother and two affected children also carry the mutation. Affected family members do not fulfil the criteria for MFS. No unaffected relatives were available for analysis. Two affected adult siblings of the mother were uncooperative. The proband's mother died unexpectedly aged 72 of undiagnosed ruptured ascending thoracic aortic aneurysm.

\section{DISCUSSION}

Analysis of the literature reveals that mutations affecting cysteines, calcium binding amino acids, or residues conserved among similar domains, are usually associated with more severe phenotypes. ${ }^{7-9}$ Considering the six different mutations reported in this study, three mutations (R122C, R240C, $\mathrm{R} 545 \mathrm{C}$ ) have already been reported, all of them involving the substitution of a cysteine for a non-conserved arginine. Mutation R122C has been reported four times,,$^{13} 1819$ in all cases the patient presenting with atypical MFS, due to the lack of serious cardiovascular manifestations. Mutation R240C has been reported twice, in a classic MFS patient ${ }^{13}$ and in an EL patient, ${ }^{14}$ reflecting the interfamilial phenotype variability. Mutation R545C has been reported twice, in patients with cardiovascular involvement. ${ }^{13}{ }^{17}$ Nevertheless, in our series, these three mutations were characterised in four patients without any serious cardiovascular involvement.

Overall, four out of seven mutations (57\%) reported in our study involved the substitution of a cysteine for a nonconserved arginine. This could be driven by the highly probable $\mathrm{C}$ to $\mathrm{T}$ transitions at $\mathrm{CpG}$ dinucleotides, but conversely arginine to cysteine mutations have been reported in only 10 of the 137 entries $(\sim 7 \%)$ of the Marfan database ${ }^{8}$ $(\mathrm{p}<0.005$, Fisher's test $)$ and in only four of the 219 entries $(\sim 2 \%)$ of the FBNl Human Gene Mutation Database, Cardiff (HGMD; http://archive.uwcm.ac.uk/uwcm/mg/search/ 127115.html $)(p<0.0001$, Fisher's test $)$. This statistically significant difference might be indicative of a recurrent type of mutation in the group of patients affected by predominant EL.

Moreover, the same type of mutation recurs in two out of four reported cases of predominant EL. ${ }^{12-14}$

Considering the three novel mutations described, C652Y causes a cysteine substitution in a cbEGF-like domain in patient MG. The severe nature of the mutation identified, the family history and the borderline aortic root dimension make long term echocardiogram follow up of all family members necessary. Could possible worsening of the condition be anticipated by the molecular diagnosis, or is this a mildly affected patient carrying an apparently severe mutation?
Mutation N164S (patient BM) causes an asparagine to serine substitution in an EGF-like domain. This type of mutation has been reported five times in the literature, ${ }^{80}$ but in all cases it involved an asparagine part of a consensus sequence for calcium binding. The mutation in this study is instead a substitution of a variable residue and is conservative, with both amino acids polar but uncharged. It is not clear if this mutation is causative.

Mutation S634P (patient NS) does not affect a conserved or invariant amino acid for cbEGF-like domains. Although it is not considered classically severe, it remains to be established what is the real effect of a non-conservative substitution of a non-polar for a polar uncharged residue. It should be noted that the proband's father could be described as a marfanoid patient, thus suggesting a case of variable penetrance.

FBNl variant $\mathrm{A} / \mathrm{G} 3963$ (patient VW) affects the second to last nucleotide of exon 31 and it has previously been reported as a polymorphism. ${ }^{21}$ However, the A residue at this position is predominant in FBNl exon "donor sequences" (56\%) and the same mutation has been observed in a patient with late onset aortic aneurysm, EL and marfanoid build (P Johnson, personal communication), where it affects the splicing of exon 31 through activation of a cryptic splice site. The unavailability of RNA from patient VW does not permit further analysis.

IVS6 del T -26 (patient IBP) and IVS62 A+8 to C (patient $\mathrm{MZ}$ ) are other FBN1 variants observed in this study. They represent rare polymorphisms already reported in the literature. ${ }^{142}$

In this study we report a success rate of $7 / 11$ (63\%) in identifying causative or putative FBN1 mutations in patients affected by predominant EL with marfanoid habitus. These results are within the $23 \%-86 \%$ range of mutation identification rate in MFS and MFS related patients in recent investigations. . $^{14161721}$ These results confirm that SSCA, although not $100 \%$ successful, is a suitable method and a viable alternative to direct sequencing for mutations identification, because of its low cost, simplicity, and efficiency, once the optimal conditions have been established.

Although the group studied is small, it is the largest predominant EL consecutive series yet reported. All mutations described in this paper are in the first 15 exons of the gene, while the Marfan database mutations ${ }^{8}$ are distributed over the gene length, with just $21 / 137$ (15\%) identified in the same region (Fig l) $(\mathrm{p}<0.0001$, Fisher's test). This statistically significant difference is maintained even when compared with the HGMD ( $p<0.0001$, Fisher's test), where 31/219 mutations ( $14 \%)$ are within the first 15 exons.

The N-terminal domains of fibrillin- 1 have a role in directing the formation of dimer intermediates which aggregate to form the functional microfibril. ${ }^{23}$ Clustering of the mutations identified in this study at the $\mathrm{N}$-terminus may underline its 
different role when compared with the middle region of the protein. In our series of patients a relatively mild phenotype, with late onset of stable cardiovascular features in some cases has been observed. In contrast, mutations in the central region, which includes the neonatal cluster of exons 24-32, are usually associated with more severe phenotypes. ${ }^{10}$ Furthermore, it has been reported that mutations in the C-terminal end of the protein could also be linked to less severe phenotypes ${ }^{20}$ thus stressing the possibility that involvement of different regions of the FBNI gene might be partially responsible for phenotype variability. Further studies of patients representing the mild end of the MFS spectrum will help to clarify this issue.

Meanwhile, since a tendency to late onset aortic dilatation and/or dissection is an occasional feature, it is recommended that patients with predominant EL be screened with echocardiography initially and at regular intervals throughout their lifetime.

\section{ACKNOWLEDGEMENTS}

We are grateful to Miss Zoë Adams, Dr Phil Johnson, and Dr Jan Poloniecki for their help and contribution. We wish to thank the Marfan Trust, the Bluff Field Charitable Trust, the Ivemark Research Fund (in memory of Miles Parker), the London Law Trust, St George's Hospital Medical School and NHS Trust, and the Marfan Association UK.

PC and ALE contributed equally to this work.

\section{Authors' affiliations}

P Comeglio, A L Evans, Sonalee Laboratory for Marfan Syndrome and Related Disorders, St George's Hospital Medical School, London, UK

G Brice, A H Child, Department of Cardiological Sciences, St George's Hospital Medical School, London, UK

R J Cooling, Vitreoretinal Surgical Unit, Moorfields Eye Hospital, London, UK

Correspondence to: Dr Paolo Comeglio, Sonalee Laboratory for Marfan Syndrome and Related Disorders, Department of Cardiological Sciences, St George's Hospital Medical School, Cranmer Terrace, London

SW17 ORE, UK; p.comeglio@sghms.ac.uk

Accepted for publication 8 July 2002

\section{REFERENCES}

1 Dietz HC, Pyeritz RE. Mutations in the human gene for fibrillin-1 (FBN1) in the Marfan syndrome and related disorders. Hum Mol Genet 1995;4:1799-809.

2 Tsipouras P, Del Mastro R, Sarfarazi M, et al. Genetic linkage of the Marfan syndrome, ectopia lentis, and congenital contractural arachnodactyly to the fibrillin genes on chromosomes 15 and 5. N Engl J Med 1992;326:905-9.
3 De Paepe A, Devereux RB, Dietz HC et al. Revised diagnostic criteria for the Marfan syndrome. Am J Med Genet 1996;62:417-26.

4 Lee B, Godfrey M, Vitale E, et al. Linkage of Marfan syndrome and a phenotypically related disorder to two different fibrillin genes. Nature 1991;352:330-4.

5 Pereira L, D'Alessio M, Ramirez F, et al. Genomic organization of the sequence coding for fibrillin, the defective gene product in Marfan syndrome. (Erratum: Hum Mol Genet 1993;2:1762). Hum Mol Genet 1993;2:961-8.

6 Biery NJ, Eldadah ZA, Moore CS, et al. Revised genomic organization of FBN1 and significance for regulated gene expression. Genomics 1999;56:70-7.

7 Hayward C, Brock DJH. Fibrillin-1 mutations in Marfan syndrome and other type-1 fibrillinopathies. Hum Mutat 1997;10:415-23.

8 Collod-Beroud G, Beroud C, Ades L, et al. Marfan Database (third edition): new mutations and new routines for the software. Nucleic Acids Res 1998;26:229-33.

9 Robinson PN, Godfrey M. The molecular genetics of Marfan syndrome and related microfibrillopathies. J Med Genet 2000;37:9-25.

10 Puinam EA, Cho M, Zinn AB, et al. Delineation of the Marfan phenotype associated with mutations in exons 23-32 of the FBN1 gene. Am J Med Genet 1996;62:233-42.

11 Tiecke F, Katzke S, Booms $P$, et al. Classic, atypically severe and neonatal Marfan syndrome: twelve mutations and genotype-phenotype correlations in FBN1 exons 24-40. Eur J Hum Genet 2001;9:13-21.

12 Lönnqvist L, Child A, Kainulainen K, et al. A novel mutation of the fibrillin gene causing ectopia lentis. Genomics 1994;19:573-6.

13 Loeys B, Nuytinck L, Delvaux I, et al. Genotype and phenotype analysis of 171 patients referred for molecular study of the fibrillin-1 gene FBN1 because of suspected Marfan syndrome. Arch Intern Med 2001:161:2447-54.

14 Körkkö J, Kaitila I, Lönnqvist L, et al. Sensitivity of conformation sensitive gel electrophoresis in detecting mutations in Marfan syndrome and related conditions. J Med Genet 2002;39:34-41.

15 Comeglio P, Evans AL, Brice GW, et al. Detection of six novel FBN1 mutations in British patients affected by Marfan syndrome. (Erratum: Hum Mut $2001 ; 18: 546-7)$. Hum Mut 2001; 18:251.

16 Nijbroek G, Sood S, McIntosh I, et al. Fifteen novel FBNI mutations causing Marfan syndrome detected by heteroduplex analysis of genomic amplicons. Am J Hum Genet 1995;57:8-21

17 Hayward C, Porteous ME, Brock DJH. Mutation screening of all 65 exons of the fibrillin-1 gene in 60 patients with Marfan syndrome: report of 12 novel mutations. Hum Mutat 1997; 10:280-9.

18 Stahl-Hallegren C, Ukkonen T, Kainulainen K, et al. An extra cysteine in one of the non-calcium-binding Epidermal Growth Factor-like motifs of the FBN1 polypeptide is connected to a novel variant of Marfan syndrome. J Clin Invest 1994:94:709-13.

19 Black C, Withers AP, Gray JR, et al. Correlation of a recurrent FBN mutation (R 122C) with an atypical familial Marfan syndrome phenotype. Hum Mutat 1998; S1:S198-200.

20 Palz M, Tiecke F, Booms $\mathrm{P}$, et al. Clustering of mutations associated with mild Marfan-like phenotypes in the $3^{\prime}$ region of FBN1 suggests a potential genotype-phenotype correlation. Am J Med Genet 2000:91:212-21.

21 Halliday D, Hutchinson S, Kettle S, et al. Molecular analysis of eight mutations in FBN1. Hum Genet 1999; 105:587-97.

22 Liu Wo, Oefner PJ, Qian C, et al. Denaturing HPLC-identified novel FBN1 mutations, polymorphisms, and sequence variants in Marfan syndrome and related connective tissue disorders. Genet Test 1997; 1:237-42.

23 Trask TM, Ritty TM, Broekelmann T, et al. N-terminal domains of fibrillin 1 and fibrillin 2 direct the formation of homodimers: a possible first step in microfibril assembly. Biochem J 1999;340:693-701. 